\title{
Proposta de protocolo para solicitação de exames de imagens odontológicas
}

\author{
Recommended protocol for requesting dental imaging exams \\ Protocolo sugerido para solicitar exámenes por imágenes dentales
}

Recebido: 27/05/2021 | Revisado: 06/06/2021 | Aceito: 08/06/2021 | Publicado: 23/06/2021

Maria Isabel de Oliveira e Britto Villalobos
ORCID: https://orcid.org/0000-0002-8675-9186
São Leopoldo Mandic, Brasil
E-mail: contato@ misabel.com.br
Joelma de Fátima Martorano
ORCID: https://orcid.org/0000-0002-1960-4854
E-mail: joelma.martonaro@gmail.com
Regina Juhás
Fundação para o Desenvolvimento Cientifico e Tecnologico da Odontologia, Brasil
ORCID: https://orcid.org/0000-0003-0660-6532
Fundação para o Desenvolvimento Cientifico e Tecnológico da Odontologia, Brasil
E-mail: rjuhas@uol.com.br
Emerson Nakao
ORCID: https://orcid.org/0000-0003-1067-8539
Fundação para o Desenvolvimento Cientifico e Tecnológico da Odontologia, Brasil
E-mail: enakao@icloud.com

\section{Resumo}

A partir dos artigos encontrados, os autores confeccionaram uma proposta de diretrizes de utilização de exames baseada em evidências científicas, para os exames de imagem mais utilizados na odontologia. A seleção dos artigos se deu com base nos seguintes critérios de inclusão: trabalhos publicados entre janeiro/2000 e dezembro/2020, revisões de literatura (sistemática, integrativa ou descritiva), principal temática relacionada a indicação de radiografias odontológicas e protocolos de indicação de exames imaginológicos utilizados em odontologia. Colaborando com o ecossistema de saúde pública e suplementar e analisando o uso racional dos recursos, o presente trabalho também tem por objetivo auxiliar entidades e profissionais da área a procederem previamente a uma análise baseada em consensos científicos que consideram faixa etária e situação clínica do paciente para solicitação de exames de imagens odontológicas. Antes da solicitação de qualquer exame complementar, recomenda-se criteriosa avaliação clínica. Esse processo permite a adoção das melhores práticas odontológicas e a tomada de decisão clínica baseada em evidências que inclui não somente a melhor proposta de tratamento, mas também a melhor oportunidade de tratamento e seleção de exames de imagem. A proposta de Diretrizes para exames de imagem em odontologia pode ser utilizada por todos os atores envolvidos na prestação de serviços odontológicos e, devido ao avanço da tecnologia e visando a manutenção de acordo com as normas vigentes, a atualização de dados a cada dois anos.

Palavras-chave: Radiologia; Administração da prática odontológica; Legislação odontológica; Odontologia baseada em evidências.

\begin{abstract}
From the articles found, the authors made a proposal for guidelines for the use of exams based on scientific evidence, for the most used imaging exams in dentistry. The selection of articles was based on the following inclusion criteria: papers published between January/2000 and December/2020, literature reviews (systematic, integrative or descriptive), main theme related to the indication of dental radiographs and protocols for the indication of exams imaging devices used in dentistry. Collaborating with the public and supplementary health ecosystem and analyzing the rational use of resources, this work also aims to help entities and professionals in the area to carry out a prior analysis based on scientific consensus that considers the patient's age group and clinical situation for ordering dental imaging exams. Before requesting any complementary exam, a careful clinical evaluation is recommended. This process allows the adoption of the best dental practices and evidence-based clinical decision-making that includes not only the best treatment proposal, but also the best treatment opportunity and imaging exam selection. The proposed Guidelines for Image Examinations in Dentistry can be used by all actors involved in the provision of dental services and, due to the advancement of technology and aiming at maintaining in accordance with current standards, data update every two years.
\end{abstract}

Keywords: Radiology; Delivery of health care; Legislation, dental; Evidence-based dentistry. 


\begin{abstract}
Resumen
A partir de los artículos encontrados, los autores realizaron una propuesta de pautas para el uso de pruebas basadas en evidencia científica, para las pruebas de imagen más utilizadas en odontología. La selección de artículos se basó en los siguientes criterios de inclusión: artículos publicados entre enero / 2000 y diciembre / 2020, revisiones de la literatura (sistemática, integradora o descriptiva), tema principal relacionado con la indicación de radiografías dentales y protocolos para la indicación de exámenes de imagen. dispositivos utilizados en odontología. Colaborando con el ecosistema sanitario público y complementario y analizando el uso racional de los recursos, este trabajo también tiene como objetivo ayudar a las entidades y profesionales del área a realizar un análisis previo basado en el consenso científico que considere el grupo de edad del paciente y su situación clínica para la realización de pedidos dentales. exámenes por imágenes. Antes de solicitar cualquier examen complementario, se recomienda una cuidadosa evaluación clínica. Este proceso permite la adopción de las mejores prácticas dentales y la toma de decisiones clínicas basadas en la evidencia que incluye no solo la mejor propuesta de tratamiento, sino también la mejor oportunidad de tratamiento y la selección de exámenes por imágenes. Las Directrices propuestas para los exámenes de imagen en odontología pueden ser utilizadas por todos los actores involucrados en la prestación de servicios dentales y, debido al avance de la tecnología y con el objetivo de mantener de acuerdo con los estándares actuales, la actualización de los datos cada dos años.
\end{abstract}

Palabras clave: Radiología; Prestación de atención de salud; Legislación em odontologia; Odontologia baseada em la evidencia.

\title{
1. Introdução
}

As radiografias são comumente utilizadas no exercício da Odontologia, sendo realizadas em ambiente ambulatorial com finalidade de diagnóstico (Liedke, Sping-Neto, \& Da Silveira 2014; Hwang, et al, 2018). Com o desenvolvimento da tecnologia digital, as doses de radiação foram reduzidas em até 50\% em comparação com a analógica (Candeiro, Bringel, \& Vale, 2009). Os exames radiográficos estão cada vez mais sendo solicitados e utilizados pelos profissionais da odontologia (Alves, et al, 2014; Hwang, et al, 2018) na busca por diagnóstico, doenças ocultas (aquelas que não podem ser verificadas clinicamente) e para registrar os tratamentos realizados e consequentemente compor os prontuários odontológicos, em respeito à legislação em vigor. Cabe ressaltar que as orientações de exames radiográficos devem estar alinhadas com a legislação brasileira quanto a utilização da radiação X (Alves, et al, 2014).

Os exames radiográficos em Odontologia são complementares a avaliação clínica, não apenas para o diagnóstico e elaboração do plano de tratamento, mas também para detecção de condições patológicas assintomáticas (Beneyto, Baños, Lajarín, Rushton, 2007; Oliveira, Silva, Junqueira, Oliveira, 2012). A indicação técnica para um exame radiográfico deve respeitar a individualidade do paciente, de acordo com a situação clínica apresentada. Além da correta indicação da radiografia, o profissional deve ficar atento à qualidade da imagem, preconizar a melhor técnica para cada caso, utilizar apenas as áreas de abrangências necessárias para o diagnóstico e tratamento adequados, e por fim, seguir o protocolo de biossegurança em radiologia odontológica (Oliveira, Silva, Junqueira, Oliveira, 2012; Beneyto, Baños, Lajarín, Rushton, 2014).

A resolução vigente que estabelece os requisitos sanitários para organização e funcionamento de serviços de radiologia odontológica e que regulamenta o controle de exposições decorrentes do uso de tecnologias radiológicas diagnósticas é a RDC 330. Essa resolução pontua a necessidade da seleção adequada de técnicas radiográficas visando a redução da radiação ionizante, conforme os princípios da justificação, da otimização, da limitação da dose e da prevenção de acidentes (Agência Nacional de Vigilância Sanitária, 2019). Apesar disso, não existe um consenso no Brasil (Ministério da Saúde, 2008) que oriente a indicação clínica de diagnóstico por imagem na Odontologia segundo a faixa etária e situação clínica, o que gera a realização - muitas vezes desnecessárias - desses exames que utilizam a radiação ionizante. Sendo assim, o presente trabalho tem o objetivo de apresentar uma proposta de diretrizes de utilização de exames por imagens odontológicas visando o uso racional do recurso, com consequente redução do número de solicitações consideradas não necessárias pela literatura científica atual; e esclarecer aos dentistas as corretas indicações para os exames radiográficos baseada em faixa etária e situação clínica. 


\section{Metodologia}

Foi realizada revisão de literatura baseada nos artigos encontrados nas seguintes bases de dados: PubMed, Lilacs, Cochrane e Bireme. As buscas foram feitas com os seguintes descritores em português "radiologia odontológica", "assistência à saúde", "assistência odontológica", "administração da prática odontológica", "radiografia panorâmica", "radiografia interproximal", "radiografia periapical", "diretrizes para o planejamento em saúde", "legislação odontológica" e os seguintes descritores em inglês "dental radiology", "practice management, dental", "delivery of health care", "radiography, panoramic", "radiography, bitewing", "radiography, periapical", "health planning guidelines" and "legislation, dental". A partir dos artigos encontrados, os autores confeccionaram uma proposta de diretrizes de utilização de exames baseada em evidências científicas, para os exames de imagem mais utilizados na odontologia. A seleção dos artigos se deu com base nos seguintes critérios de inclusão: trabalhos publicados entre janeiro/2000 e dezembro/2020, revisões de literatura (sistemática, integrativa ou descritiva), principal temática relacionada a indicação de radiografias odontológicas e protocolos de indicação de exames imaginológicos utilizados em odontologia.

\section{Discussão}

As imagens radiográficas auxiliam os dentistas na formulação do diagnóstico e fornecem informações essenciais, como por exemplo: lesões de cárie, reabsorções do osso alveolar, calcificações pulpares e periodontais, espaço do ligamento periodontal alargado, presença ou ausência de lâmina dura, altura óssea do rebordo alveolar, comprometimento na região de furca, lesões endoperiodontais, locais retentivos de biofilme, entre outras. Os exames imaginológicos são de grande valia para o acompanhamento da evolução dos tratamentos, auxiliam em possíveis processos judiciais e no acompanhamento da situação dos pacientes (Farman. 2007; Cerril Guarim, Genovese, American Dental Association, 2012). Cada tipo de exame radiográfico deverá ser utilizado a depender da situação apresentada pelo paciente durante o exame clínico.

Um dos exames mais solicitados pelos cirurgiões-dentistas é a radiografia panorâmica. Esse exame possui baixa dose de radiação e consegue, em apenas uma única imagem, mostrar todos os órgãos dentais e regiões adjacentes (Capote, Gonçalves, Gonçalves, Gonçalves, 2005; Villoria, et al, 2016). Por isso, para pacientes novos, alguns autores indicam que após a avaliação clínica, esse exame seja solicitado como forma de complementação da análise geral da situação do paciente e para verificação de possíveis lesões assintomáticas (Villoria, et al, 2016). Em contrapartida, esse "protocolo" não é muito bem aceito entre alguns profissionais, já que as lesões completamente assintomáticas são raras (Beneyto, Baños, Lajarín, Rushton, 2007; Sulimana, Abdelgadir, 2018; Kühnisch, et al, 2019). Os autores que defendem que esses exames não sejam solicitados como rotina afirmam que ele não deve ser realizado se houver outros métodos para análise da situação clínica do paciente. $\mathrm{O}$ exame clínico é soberano por ter larga eficácia diagnóstico quando devidamente realizado. O exame complementar, como a solicitação de radiografias panorâmicas, deve ser indicado adequadamente, a fim de não expor os pacientes a exames desnecessários (Beneyto, Baños, Lajarín, Rushton, 2007; Kühnisch, et al, 2019; Sulimana, Abdelgadir, 2018), conforme a legislação brasileira vigente, os exames de imagem devem ser solicitados respeitando os princípios da justificação, otimização, limitação da dose e da prevenção de acidentes, ou seja, devem ser realizados quando existem benefícios suficientes para o indivíduo ou para a sociedade (Agência Nacional de Vigilância Sanitária, 2019). Outros autores defendem que esse exame é um dos mais didáticos e de fácil compreensão, sendo um grande auxílio visual para apresentação e discussão do caso com os pacientes (Capote, Gonçalves, Gonçalves, Gonçalves, 2005; White Pharoah 2020).

Além da solicitação em pacientes novos, existem outras indicações em que se preconiza solicitação das radiografias panorâmicas como: dente inclusos/impactados em posição que impossibilita realização de radiografia periapical; problemas odontológicos generalizados (após análise clínica) e com bolsa periodontal superior a 6mm; avaliação de terceiros molares para planejamento cirúrgico; planejamento ortodôntico, após avaliação clínica e verificação de dentes supranumerários ou 
dentes ausentes (Capote, Gonçalves, Gonçalves, Gonçalves, 2005; Beneyto, Baños, Lajarín, Rushton, 2007; American Dental Association, 2012;Kühnisch, et al, 2019; Tsiklakis, Mitsea, Tsichlaki, Pandis, 2019).

Apesar das diversas vantagens desse exame, a radiografia panorâmica carece de detalhes e resolução de algumas estruturas, resultando em distorção e/ou sobreposição de imagens (Farman, 2007; Rushton, Horner, Worthington, 2001; Beneyto, Baños, Lajarín, Rushton, 2007). Assim, nesses casos se faz necessária a realização de radiografias intraorais localizadas que permitam melhor detalhamento dessa região, como as periapicais.

As radiografias interproximais, também conhecidas como bitewing, permitem a análise das superfícies interproximais das coroas de pré-molares e molares sem o viés da sobreposição. Essa técnica radiográfica costuma ser utilizada para novos pacientes como complemento ou não da radiografia panorâmica, e para pesquisa de cáries (proximais e incipientes). Também é indicada para avaliação de adaptação de restaurações interproximais diretas e indiretas, e análise da crista óssea alveolar. Cabe ressaltar que é um consenso entre os mais diversos autores que essas radiografias devem ser solicitadas somente após exame clínico (Sariyilmaz, Keskin, Ozcan, 2016; Villoria, Francio, Cunha, Manzi, 2016; Caballero, Arenas, Acosta, 2017).

Outros exames que também são comumente solicitadas na Odontologia, as radiografias periapicais são realizadas através de duas técnicas (bissetriz e paralelismo) para análise do órgão dentário e estruturas anatômicas vizinhas. $\mathrm{O}$ exame radiográfico é conhecido como "exame boca toda", divididos em sete regiões da maxila e sete da mandíbula, em um total de 14 filmes periapicais podendo variar de 14 a 18 radiografias quando solicitado interproximais (Castro, et al, 2017; White Pharoah 2020).

Esse tipo de técnica radiográfica somente deve ser solicitado, segundo a literatura, após a avaliação clínica e verificação de situações específicas como: doença periodontal generalizada (acometendo vários dentes), avaliação da qualidade de procedimentos restauradores diretos e indiretos e na interface implante/componente protético/integridade peri-implantar, restaurações subgengivais, cárie generalizada (acometendo vários dentes), acompanhamento de tratamentos odontológicos extensos, análise da relação entre a dentição decídua e permanente, verificação de mineralizações e nódulos pulpares, suspeita de reabsorções radiculares internas e externas, análise da qualidade da obturação do canal radicular no tratamento endodôntico, avaliação de oclusões traumáticas, suspeita de fraturas radiculares, anomalias dentárias, lesões periapicais e doenças ósseas maxilomandibulares (American Dental Association, 2012; Liedke, Spin-Neto, Da Silveira Wenzel 2014; Correia-Sousa, et al, 2015; Sariyilmaz, Keskin, Ozcan, 2016; Villoria, Francio, Cunha, Manzi, 2016; Castro, et al, 2017; Caballero, Arenas, Acosta, 2017; Zavala, Mendiburu, Lugo-Ancona, 2017).

Ainda referente ao uso de radiografias periapicais, elas devem ser consideradas como "de escolha" na avaliação endodôntica do paciente, já que essa técnica apresenta imagens mais precisas para o acompanhamento de tamanhos de lesões, auxiliando na comparação entre o a história do paciente antes, durante e após a conclusão da terapêutica endodôntica, principalmente quando o osso cortical estava envolvido (Diangelis, et al, 2012; Villoria, Francio, Cunha, Manzi, 2016). Radiografias periapicais convencionais são mais comumente solicitadas para a avaliação da região periapical, porém, a sobreposição de estruturas ósseas pode prejudicar a visualização de imagens radiolúcidas periapicais, principalmente com lesões iniciais (Villoria, Francio, Cunha, Manzi, 2016).

$\mathrm{Na}$ Europa, a odontologia é uma especialidade na área da medicina e um estudo verificou que as radiografias odontológicas estão entre as mais solicitadas da área. Essas radiografias representam um terço de todos os exames de imagens (por exemplo: radiografias, tomografias e ressonâncias magnéticas) realizados nos países que fazem parte da União Europeia (Beneyto, Baños, Lajarín, Rushton, 2007). No Brasil, em um estudo realizado em uma Faculdade em Minas Gerais, os autores verificaram que as radiografias periapicais então entre os exames mais solicitados, principalmente para avaliações de endodontia e periodontia. A radiografia interproximal aparece logo em seguida, geralmente solicitada para avaliações de lesões cariosas (Oliveira, Silva, Junqueira, Oliveira, 2012). Esses dados corroboram com a análise de solicitação de radiografias feito 
em uma Universidade em São Paulo, em que as radiografias periapicais e interproximais também foram as mais solicitadas (Gil, et al, 2005). Já em um levantamento realizado em uma Faculdade de Odontologia em Pelotas, Rio Grande do Sul, a radiografia periapical $(64,41 \%)$ apresentou a maior porcentagem de solicitação e a interproximal esteve em apenas 3,73\% dos casos (Alves, et al, 2014).

No mesmo estudo em Pelotas, a radiografia panorâmica apareceu como a segunda mais solicitada, em 31,02\% dos casos (Alves, et al, 2014). Esse tipo de exame é geralmente solicitado para avaliação de inclusão dental, problemas odontológicos generalizados ou sindrômicos e planejamento ortodôntico (Capote, Gonçalves, Gonçalves, Gonçalves, 2005; Gil, et al, 2005; Beneyto, Baños, Lajarín, Rushton, 2007; Oliveira, Silva, Junqueira, Oliveira, 2012; Kühnisch, et al, 2019; Tsiklakis, Mitsea, Tsichlaki, Pandis, N., 2019). Muitos profissionais também solicitam para análise e planejamento de reabilitação com implantes dentários, associado a tomografia computadorizada (Oliveira, Silva, Junqueira, Oliveira, 2012). Ainda sobre o número de solicitações, a radiografia panorâmica esteve em terceiro lugar entre os exames de imagem mais solicitados na área odontológica (Gil, et al, 2005; Beneyto, Baños, Lajarín, Rushton; Oliveira, Silva, Junqueira, Oliveira, 2012)

Em avaliação do perfil dos pacientes encaminhados para a realização de exames radiográficos odontológicos, considerando gênero e faixa etária, os resultados revelaram que o maior número de pacientes atendidos era jovem do gênero feminino. Ao analisar o grupo de indivíduos mais velhos (homens entre 50-59 anos e mulheres entre 60-69 anos), foi observado um maior número de exames radiográficos solicitados (Gil, 2005). Isso pode ter relação com o aumento do risco a doença periodontal em pessoas de idade mais avançada e da indicação de radiografias periapicais e interproximais para acompanhamento desses casos (Villoria, Francio, Cunha, Manzi, 2016; Caballero, Arenas, Acosta, 2017).

O estudo relacionado às Diretrizes Europeias na proteção contra radiação em radiologia dentária e desenvolvido na prática baseada em evidências e sua relevância. O método proposto para eliminar os exames radiográficos desnecessários e adoção de critérios de seleção na radiologia. Os critérios de seleção foram definidos como "descrições de condições clínicas observadas a partir de sinais e sintomas do paciente e história que identifica os pacientes que precisam de um exame radiográfico específico. Concluiu-se que através da história clínica e a necessidade de exames radiológicos, as radiografias intra-orais devem ser a primeira escolha devido melhor detalhe e menor dose de radiação (Beneyto, Baños, Lajarín, Rushton, 2007). Os países europeus seguem regulamentações para proteção contra radiações como uso de colimação circular e sistema digital bem calibrado com consequente diminuição da dose, apesar de não haver limites de dose para pacientes (Kühnisch, 2019; Tsiklakis, Mitsea, Tsichlaki, Pandis, 2019).

O Projeto Sorrir (Agência Nacional de Saúde Suplementar, 2018) realizou um estudo sobre indicação e contraindicação das radiografias panorâmica, periapical e interproximal. Concluiu-se que todo o exame radiográfico deve ser solicitado após exame clínico avaliando sinais e sintomas e que a solicitação será determinante de acordo com a queixa e o diagnóstico inicial (Agência Nacional de Saúde Suplementar, 2018) e uma mesma diretriz adotada pelo FDA (Food and Drug Administration) e pela ADA (American Dental Association) (American Dental Association, 2012; Agência Nacional de Saúde Suplementar, 2018). Essa também foi a conclusão em outros estudos realizados em crianças de 2 a 18 anos com leucemia linfoblástica aguda (LLA) em que se observou alterações na cavidade oral devido a quimioterapia. Nesses casos, o exame intraoral é de extrema importância para estabelecer o diagnóstico das lesões orais e a maioria apresentou alterações durante ou após o início da quimioterapia (Morais, 2014).

Foi realizado uma pesquisa pela Faculdade de odontologia da USP (Universidade do Estado de São Paulo) com 252 crianças, entre 3 e 6 anos, mostrando que o exame radiológico não se configura como melhor método de diagnóstico de cáries em dentes decíduos (dente de leite), devido à ocorrência de resultados falso-positivos que induziram cirurgiões dentistas a realizar intervenções desnecessárias, como em casos de imagem radiolúcida na radiografia que não evoluíram antes que ocorresse a troca da dentição na criança, chamado sobrediagnósticos. Segundo estudo, o exame clínico ou inspeção visual 
isolado (VIS) ainda é o mais recomendado para esta idade. Além de diminuir a quantidade de procedimentos odontológicos, adiam o tratamento para que a criança esteja mais madura emocionalmente. O mesmo estudo mostrou que o uso da radiografia para diagnóstico das lesões é o mais adotado em consultórios odontológicos, considerando que a cárie infantil em dentes decíduos atinge 500 milhões de crianças no mundo. Como resultado dessa pesquisa os falsos positivos do grupo de radiografias foram dez vezes maiores que o grupo de exame clínico visual e tiveram $30 \%$ a mais de tratamento de intervenções do que aquelas que fizeram somente o exame clínico visual (Mendes, et al, 2016; Pontes, 2020).

A Academia Europeia de Odontopediatria (AEO) realizou estudos com três grupos de trabalho compostos por especialistas convidados e delegados nomeados pela AEO, trata-se de práticas clínicas e prescrição de radiografias dentárias para crianças e adolescentes. A prescrição foi considerada para casos de detecção e avaliação de cárie, diagnóstico de infecções dentárias, trauma dentário, anomalias dentárias, distúrbios do desenvolvimento ou achados patológicos. A dosagem de radiação utilizada durante os exames de radiografias odontológicas é relativamente baixa quando comparada aos exames de radiografias médicas. Porém, como o diagnóstico radiográfico dentário representa quase $25 \%$ de todos os exames radiológicos solicitados, deve ser dada uma atenção especial a este tipo de exame em termos de proteção radiológica. Na odontopediatria, assim como em todas as especialidades na Odontologia, se baseia no princípio ALARA - as low as reasonably achievable que significa: tão baixo quanto razoavelmente possível, que consiste em reduzir a dose de exposição (Farman, 2007; Villoria, Francio, Cunha, Manzi, 2016; Kühnisch, 2019; Tsiklakis, Mitsea, Tsichlaki, Pandis, 2019).

Apesar da segurança dos exames radiográficos odontológicos, isso não significa que não há necessidade de diretriz de utilização para uso racional das radiografias. Conforme a legislação vigente de diretrizes básicas de proteção radiológica, a RDC (Resolução da Diretoria Colegiada) no 330, para a realização de exames radiográficos, devem ser observados os princípios da justificação, otimização, limitação da dose e prevenção de acidentes. Isso visa a garantia da exposição segura do paciente aos riscos inerentes de cada tecnologia e manutenção da qualidade esperada das imagens e procedimentos. Ainda segundo o artigo 44 da RDC 330: "Art. 44. As exposições médicas de pacientes devem ser otimizadas ao valor mínimo necessário à obtenção do objetivo radiológico, bem como ser compatíveis com os padrões aceitáveis de qualidade de imagem, devendo-se considerar, no processo de otimização de exposições médicas: I - a seleção adequada de técnicas, equipamentos e acessórios; II - os processos de trabalho; III - a garantia da qualidade; IV - os níveis de referência de diagnóstico para pacientes adultos e pediátricos; e V - as restrições de dose para indivíduo que colabore conscientemente, de livre vontade e fora do contexto de sua atividade profissional, no apoio e conforto de um paciente, durante a realização do procedimento radiológico" (Agência Nacional de Vigilância Sanitária, 2019).

Foram realizados estudos sobre os critérios de seleção do FDA com objetivo de estabelecer práticas atuais em relação aos exames radiográficos: panorâmica, periapical e interproximal. Apesar de a radiografia periapical ser mais utilizada do que a radiografia panorâmica, os critérios de seleção da FDA/ADA considera a radiografia panorâmica uma excelente ferramenta de diagnóstico em pacientes assintomáticos permitindo uma visão geral das estruturas dentoalveolares (exame de triagem geral), também oferecendo como vantagens: redução da dose de radiação, menor custo, imagem de uma área maior com radiografias intraorais complementares para verificar os achados clínicos para cada um dos pacientes (Farman, A. G., 2007; American Dental Association, 2012).

\section{Proposta de Protocolo de Solicitações de Exames de Imagens Odontológicas}

Com base na revisão da literatura e discussão de diversos trabalhos encontrados, os autores elaboraram uma proposta de protocolo de solicitação de exames de imagens odontológicas. Inicialmente, o cirurgião-dentista deverá realizar o exame clínico de acordo com a queixa principal do paciente. Essa avaliação será determinante para o tratamento e solicitação de exames complementares. Através das informações colhidas como: início da queixa, durabilidade, como evoluiu e 
características da dor se houver. A história médica pregressa é importante para pesquisa de patologias atuais ou passadas, associada ou não a queixa principal, como também hábitos de vida como: atividade física, tabagismo, sedentarismo alcoolismo. Também deve ser realizado o exame físico, que consiste na análise de sinais e sintomas clínicos divididos em inspeção para identificação de alterações que possam sugerir patologias, palpação para identificar alterações de forma, percussão de pequenos e leves golpes que através do som podemos identificar alterações patológicas e a ausculta que é semelhante a percussão só que com uso de aparelhos para este fim. (Marsi, Mengue, Bertini, Cabral, Almeida, 2009).

Devido ao avanço da tecnologia e visando a manutenção desse protocolo conforme as normas vigentes, existe um planejamento para atualização desses dados a cada dois anos. Baseados no protocolo da ADA (American Dental Association, 2012), dividimos os critérios de indicação por idade. (Tabela 1)

Tabela 1. Divisão dos grupos por faixa etária.

\begin{tabular}{|c|c|}
\hline Grupo & Idade \\
\hline Grupo 1 & $\begin{array}{c}<6 \text { ANOS } \\
\begin{array}{c}\text { Crianças com dentição decídua (antes da erupção do primeiro molar } \\
\text { permanente) }\end{array} \\
\end{array}$ \\
\hline Grupo 2 & $\begin{array}{l}\qquad 6 \text { a } 12 \text { anos } \\
\text { Crianças com dentição mista (após erupção do primeiro molar permanente até a } \\
\text { erupção do segundo molar permanente) }\end{array}$ \\
\hline Grupo 3 & $\begin{array}{c}12 \text { a } 30 \text { anos } \\
\text { Adolescentes com dentição permanente (antes da erupção dos terceiros } \\
\text { molares) }\end{array}$ \\
\hline Grupo 4 & $\begin{array}{c}>30 \text { anos } \\
\text { Adultos, dentados ou parcialmente edêntulos } \\
\end{array}$ \\
\hline Grupo 5 & Edêntulos total \\
\hline
\end{tabular}

Fonte: Autores. 
Tabela 2. Proposta de protocolo de solicitação de exames de imagens odontológicas em situações gerais na Odontologia

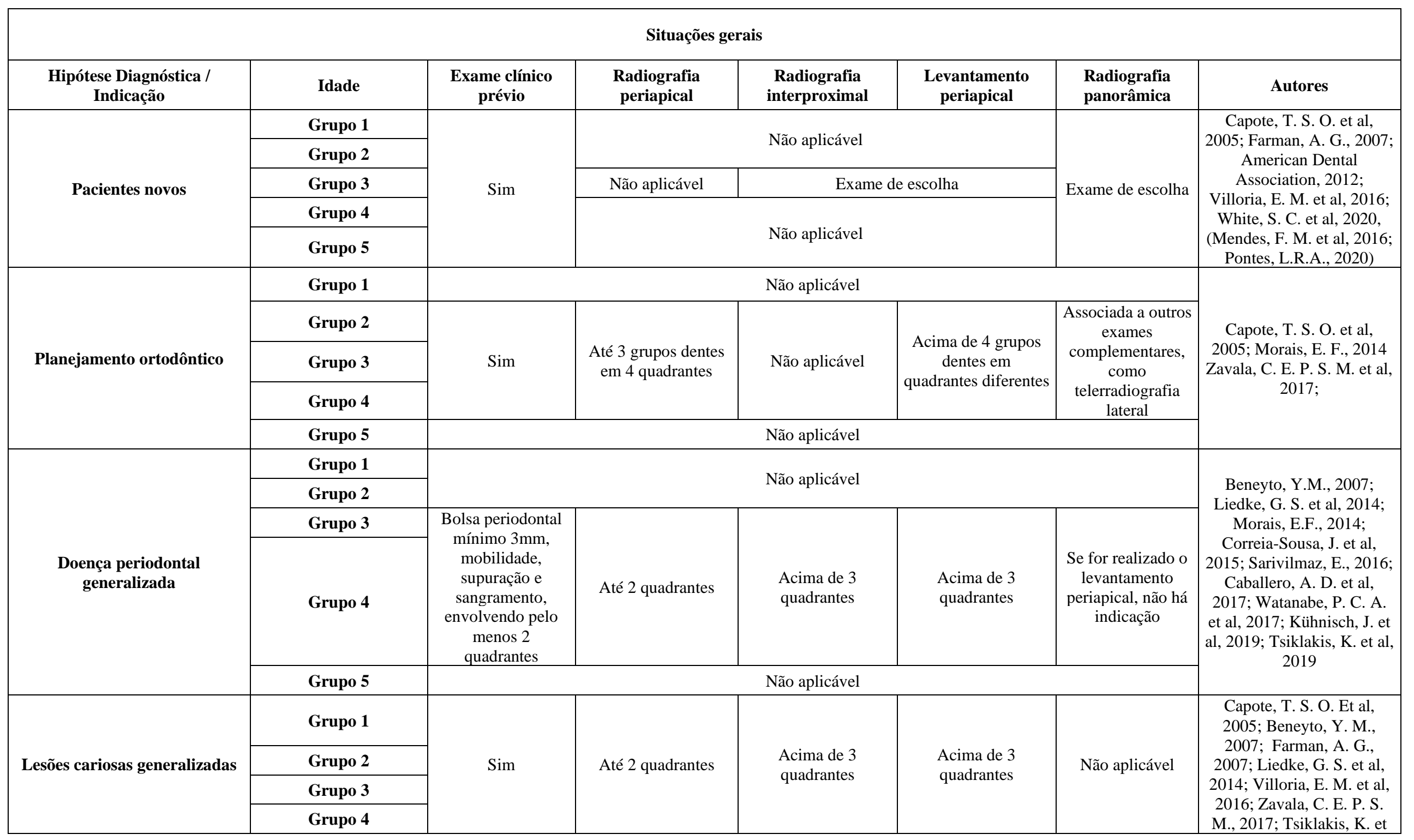


Research, Society and Development, v. 10, n. 7, e33210716573, 2021

(CC BY 4.0) | ISSN 2525-3409 | DOI: http://dx.doi.org/10.33448/rsd-v10i7.16573

\begin{tabular}{|c|c|c|c|c|c|c|c|}
\hline & Grupo 5 & & & Não aplicável & & & $\begin{array}{l}\text { al, 2019, (Mendes, F. M. et } \\
\text { al, 2016; Pontes, L.R.A., }\end{array}$ \\
\hline \multirow{5}{*}{$\begin{array}{l}\text { Excesso ou falta de materiais } \\
\text { restauradores }\end{array}$} & Grupo 1 & \multirow{4}{*}{$\begin{array}{l}\text { Avaliação clínica e } \\
\text { verificação de } \\
\text { desadaptação nas } \\
\text { faces } \\
\text { interproximais }\end{array}$} & \multirow{4}{*}{ Até 2 quadrantes } & \multirow{4}{*}{ Até 2 quadrantes } & \multirow{4}{*}{$\begin{array}{l}\text { Acima de } 3 \\
\text { quadrantes }\end{array}$} & \multirow{4}{*}{ Não aplicável } & \multirow{5}{*}{ Liedke, G. S. et al, 2014} \\
\hline & Grupo 2 & & & & & & \\
\hline & Grupo 3 & & & & & & \\
\hline & Grupo 4 & & & & & & \\
\hline & Grupo 5 & \multicolumn{5}{|c|}{ Não aplicável } & \\
\hline \multirow{5}{*}{$\begin{array}{l}\text { Avaliação cronológica das } \\
\text { erupções dentais }\end{array}$} & Grupo 1 & \multirow{3}{*}{$\begin{array}{l}\text { Erupção tardia e } \\
\text { histórico de } \\
\text { supranumerários na } \\
\text { dentição decídua }\end{array}$} & \multirow{3}{*}{ Até 2 quadrantes } & \multirow{3}{*}{ Até 2 quadrantes } & \multirow{3}{*}{$\begin{array}{l}\text { Se for realizado o } \\
\text { levantamento } \\
\text { periapical, não há } \\
\text { indicação }\end{array}$} & \multirow{3}{*}{$\begin{array}{l}\text { Acima de } 3 \\
\text { quadrantes }\end{array}$} & \multirow{5}{*}{$\begin{array}{l}\text { Capote, T. S. O. et al, } \\
\text { 2005; Kühnisch, J., et al, } \\
\text { 2019; Tsiklakis, K. et al, } \\
2019\end{array}$} \\
\hline & Grupo 2 & & & & & & \\
\hline & Grupo 3 & & & & & & \\
\hline & Grupo 4 & \multirow{2}{*}{\multicolumn{5}{|c|}{ Não aplicável }} & \\
\hline & Grupo 5 & & & & & & \\
\hline \multirow{5}{*}{$\begin{array}{l}\text { Acompanhamento de } \\
\text { tratamento com má-formaçoses } \\
\text { congênitas/má-oclusão }\end{array}$} & Grupo 1 & \multirow{4}{*}{ Sim } & \multirow{4}{*}{$\begin{array}{l}3 \text { periapicais de } \\
\text { canino a canino } \\
\text { superior }\end{array}$} & \multirow{4}{*}{ Não aplicável } & \multirow{4}{*}{ Não aplicável } & \multirow{4}{*}{ Sim } & \multirow{5}{*}{$\begin{array}{l}\text { Beneyto, Y. M. et al, 2007; } \\
\text { Sousa, A. A. et al, 2016; } \\
\text { Watanabe, P. C. A. et al, } \\
\text { 2017; Kühnisch, J. et al, } \\
2019\end{array}$} \\
\hline & Grupo 2 & & & & & & \\
\hline & Grupo 3 & & & & & & \\
\hline & Grupo 4 & & & & & & \\
\hline & Grupo 5 & \multicolumn{5}{|c|}{ Não aplicável } & \\
\hline
\end{tabular}

Fonte: Autores. 
Research, Society and Development, v. 10, n. 7, e33210716573, 2021

(CC BY 4.0) | ISSN 2525-3409 | DOI: http://dx.doi.org/10.33448/rsd-v10i7.16573

Tabela 3. Proposta de protocolo de solicitação de exames de imagens odontológicas em casos de Endodontia.

\begin{tabular}{|c|c|c|c|c|c|c|c|}
\hline \multicolumn{8}{|c|}{ Casos de Endodontia } \\
\hline $\begin{array}{c}\text { Hipótese Diagnóstica / } \\
\text { Indicação }\end{array}$ & Idade & $\begin{array}{l}\text { Exame clínico } \\
\text { prévio }\end{array}$ & $\begin{array}{c}\text { Radiografia } \\
\text { periapical }\end{array}$ & $\begin{array}{c}\text { Radiografia } \\
\text { interproximal }\end{array}$ & $\begin{array}{c}\text { Levantamento } \\
\text { periapical }\end{array}$ & $\begin{array}{l}\text { Radiografia } \\
\text { panorâmica }\end{array}$ & Autores \\
\hline \multirow{5}{*}{$\begin{array}{l}\text { Mineralizações e nódulos } \\
\text { pulpares }\end{array}$} & Grupo 1 & \multirow{2}{*}{\multicolumn{5}{|c|}{ Não aplicável }} & \multirow{10}{*}{$\begin{array}{l}\text { Beneyto, Y. M. et al, 2007; } \\
\text { Diangelis, A. J. et al, 2012; } \\
\text { Correia-Sousa, J. et al, } \\
\text { 2015; Kühnisch, J. et al, } \\
2019\end{array}$} \\
\hline & Grupo 2 & & & & & & \\
\hline & Grupo 3 & \multirow{2}{*}{$\begin{array}{c}\text { Sensibilidade local } \\
\text { e durante a } \\
\text { mastigação } \\
\end{array}$} & \multirow{2}{*}{$\begin{array}{l}\text { Até } 4 \text { dentes no } \\
\text { mesmo quadrante }\end{array}$} & \multirow{2}{*}{ Não aplicável } & \multirow{2}{*}{$\begin{array}{l}\text { Acima de } 5 \text { dentes } \\
\text { em quadrantes } \\
\text { diferentes }\end{array}$} & \multirow{2}{*}{ Não aplicável } & \\
\hline & Grupo 4 & & & & & & \\
\hline & Grupo 5 & & & Não aplicável & & & \\
\hline \multirow{5}{*}{$\begin{array}{l}\text { Reabsorções radiculares } \\
\text { internas e externas (local) }\end{array}$} & Grupo 1 & \multirow{2}{*}{\multicolumn{5}{|c|}{ Não aplicável }} & \\
\hline & Grupo 2 & & & & & & \\
\hline & Grupo 3 & \multirow{2}{*}{$\begin{array}{c}\text { Sensibilidade local } \\
\text { e durante a } \\
\text { mastigação } \\
\end{array}$} & \multirow{2}{*}{\multicolumn{2}{|c|}{ Até 4 dentes no mesmo quadrante }} & \multirow{2}{*}{$\begin{array}{c}\text { Acima de } 5 \text { dentes } \\
\text { em quadrantes } \\
\text { diferentes } \\
\end{array}$} & \multirow{2}{*}{ Não aplicável } & \\
\hline & Grupo 4 & & & & & & \\
\hline & Grupo 5 & & & Não aplicável & & & \\
\hline \multirow{5}{*}{$\begin{array}{l}\text { (Re)tratamento endodôntico e } \\
\text { acompanhamento do } \\
\text { tratamento }\end{array}$} & Grupo 1 & \multirow{2}{*}{\multicolumn{5}{|c|}{ Não aplicável }} & \multirow{5}{*}{$\begin{array}{l}\text { Capote, T. S.et al, 2005; } \\
\text { Beneyto, Y. M. et al, 2007; } \\
\text { Correia-Sousa, J. et al, } \\
\text { 2015; Villoria, E. M. et al, } \\
\text { 2016; Sarivilmaz, E. et al, } \\
\text { 2016; Cabellero, A. D. et } \\
\text { al, 2017; Zavala, C. E. P. } \\
\text { S. M. et al, 2017 }\end{array}$} \\
\hline & Grupo 2 & & & & & & \\
\hline & Grupo 3 & \multirow{2}{*}{$\begin{array}{c}\text { Presença de dor, } \\
\text { durante o } \\
\text { tratamento } \\
\text { endodôntico e após } \\
\text { para verificação da } \\
\text { qualidade } \\
\end{array}$} & \multirow[b]{2}{*}{$\begin{array}{l}\text { Até } 4 \text { dentes no } \\
\text { mesmo quadrante }\end{array}$} & \multirow[b]{2}{*}{ Não aplicável } & \multirow[b]{2}{*}{$\begin{array}{c}\text { Acima de } 5 \text { dentes } \\
\text { em quadrantes } \\
\text { diferentes }\end{array}$} & \multirow[b]{2}{*}{$\begin{array}{l}\text { Se for realizado o } \\
\text { levantamento } \\
\text { periapical, não há } \\
\text { indicação }\end{array}$} & \\
\hline & Grupo 4 & & & & & & \\
\hline & Grupo 5 & \multicolumn{5}{|c|}{ Não aplicável } & \\
\hline \multirow{5}{*}{ Fraturas radiculares } & Grupo 1 & \multicolumn{5}{|c|}{ Não aplicável } & \multirow{5}{*}{$\begin{array}{l}\text { Capote, T. S. O. et al, } \\
\text { 2005; Beneyto, Y. M. et al, } \\
\text { 2007; Diangelis, A. J. et al, } \\
\text { 2012; Liedke, G. S. et al, } \\
\text { 2014; Caballero, A. D. et } \\
\text { al, 2017; Zavala, C. E. P. } \\
\text { S. M., } 2017\end{array}$} \\
\hline & Grupo 2 & & Até 3 dentes no & & & & \\
\hline & Grupo 3 & & (OBS.: Em caso de & & & & \\
\hline & Grupo 4 & Sim & $\begin{array}{c}\text { fraturas } \\
\text { horizontais/oblíquas, } \\
\text { sugere-se Técnica de } \\
\text { Clark - } 3 \text { radiografias } \\
\text { periapicais } \\
\text { individuais) } \\
\end{array}$ & Não aplicável & & Não aplicável & \\
\hline & Grupo 5 & \multicolumn{5}{|c|}{ Não aplicável } & \\
\hline
\end{tabular}

Fonte: Autores. 
Research, Society and Development, v. 10, n. 7, e33210716573, 2021

(CC BY 4.0) | ISSN 2525-3409 | DOI: http://dx.doi.org/10.33448/rsd-v10i7.16573

Tabela 4. Proposta de protocolo de solicitação de exames de imagens odontológicas em casos de Cirurgia.

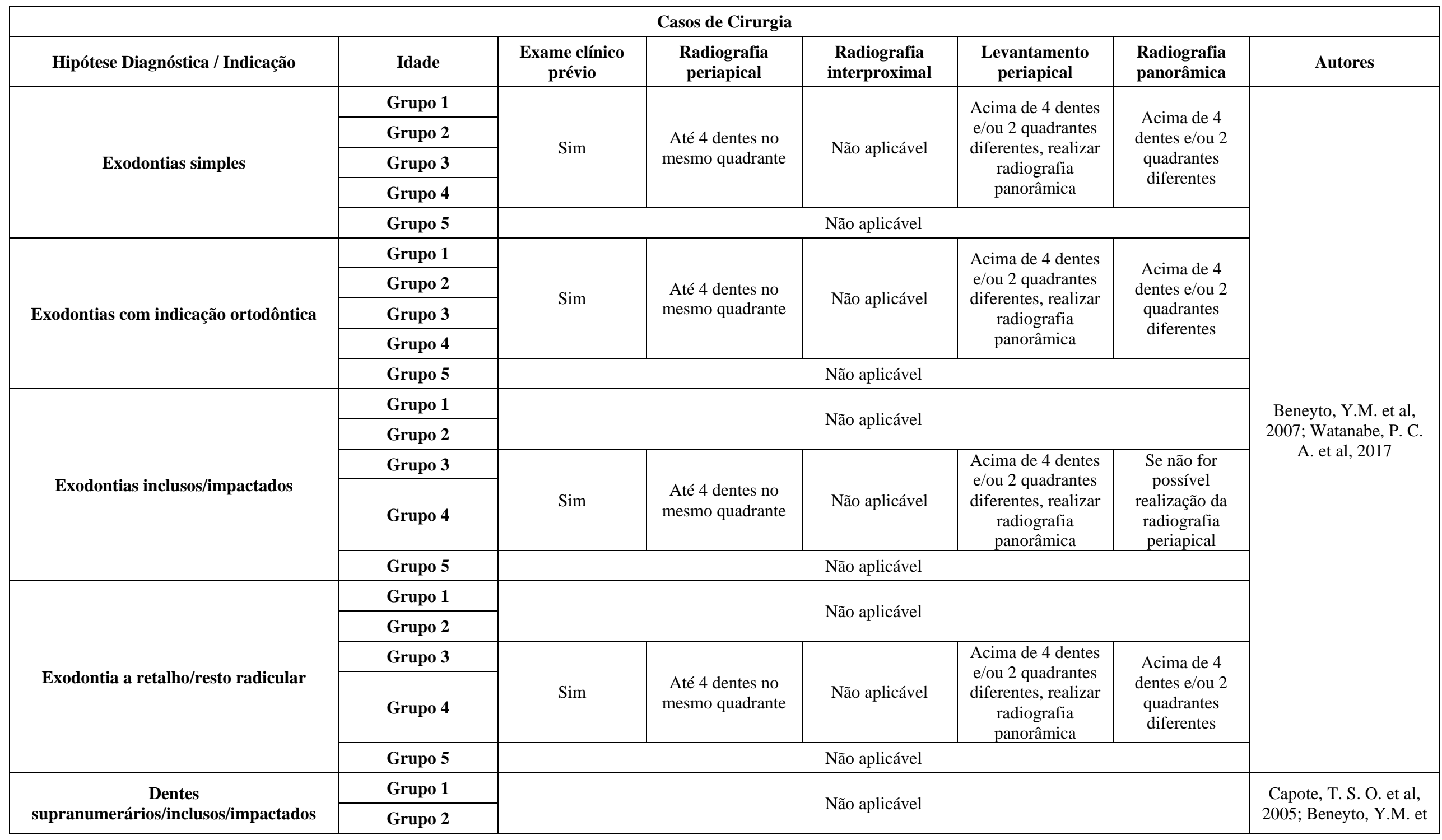


Research, Society and Development, v. 10, n. 7, e33210716573, 2021

(CC BY 4.0) | ISSN 2525-3409 | DOI: http://dx.doi.org/10.33448/rsd-v10i7.16573

\begin{tabular}{|c|c|c|c|c|c|c|c|}
\hline & Grupo 3 & $\begin{array}{l}\text { Erupção tardia e } \\
\text { histórico de } \\
\text { supranumerários } \\
\text { na dentição } \\
\text { decídua }\end{array}$ & Até 2 quadrantes & Não aplicável & $\begin{array}{l}\text { Se for realizado o } \\
\text { levantamento } \\
\text { periapical, não há } \\
\text { indicação }\end{array}$ & $\begin{array}{l}\text { Acima de } 3 \\
\text { quadrantes }\end{array}$ & $\begin{array}{l}\text { al, 2007; Watanabe, P. } \\
\text { C. A. et al, } 2017\end{array}$ \\
\hline & Grupo 4 & \multirow{2}{*}{\multicolumn{5}{|c|}{ Não aplicável }} & \\
\hline & Grupo 5 & & & & & & \\
\hline \multirow{5}{*}{ Lesões intra-ósseas } & Grupo 1 & \multirow{5}{*}{$\begin{array}{l}\text { Pode ser } \\
\text { assintomática. } \\
\text { Solicitar em caso } \\
\text { de sinais clínicos } \\
\text { após avaliação do } \\
\text { Estomatologista }\end{array}$} & \multirow{5}{*}{$\begin{array}{c}\text { Até } 3 \text { dentes em até } \\
2 \text { quadrantes }\end{array}$} & \multirow{5}{*}{ Não aplicável } & \multirow{5}{*}{$\begin{array}{l}\text { Se for realizado o } \\
\text { levantamento } \\
\text { periapical, não há } \\
\text { indicação }\end{array}$} & \multirow{5}{*}{$\begin{array}{l}\text { Exame de } \\
\text { escolha }\end{array}$} & \multirow{5}{*}{$\begin{array}{l}\text { Farman, A. G., 2007; } \\
\text { Morais, E. F. et al, 2014; } \\
\text { Villoria, E. M. et al, } \\
\text { 2016; Watanabe, P. C. } \\
\text { A. et al, 2017 }\end{array}$} \\
\hline & Grupo 2 & & & & & & \\
\hline & Grupo 3 & & & & & & \\
\hline & Grupo 4 & & & & & & \\
\hline & Grupo 5 & & & & & & \\
\hline \multirow{5}{*}{$\begin{array}{l}\text { Avaliação e acompanhamento de traumas } \\
\text { e fraturas bucomaxilofaciais }\end{array}$} & Grupo 1 & \multirow{5}{*}{ Sim } & \multirow{5}{*}{ Não aplicável } & \multirow{5}{*}{ Não aplicável } & \multirow{5}{*}{ Não aplicável } & \multirow{5}{*}{$\begin{array}{l}\text { Exame de } \\
\text { escolha }\end{array}$} & \multirow{5}{*}{$\begin{array}{l}\text { Capote, T. S. O. et al, } \\
\text { 2005; Villoria, E. M. et } \\
\text { al, 2016; Watanabe, P. } \\
\text { C. A. et al, 2017; } \\
\text { Kühnisch, J. et al, 2019; } \\
\text { Tsiklakis, K. et al, } 2019\end{array}$} \\
\hline & Grupo 2 & & & & & & \\
\hline & Grupo 3 & & & & & & \\
\hline & Grupo 4 & & & & & & \\
\hline & Grupo 5 & & & & & & \\
\hline
\end{tabular}

Fonte: Autores. 
Research, Society and Development, v. 10, n. 7, e33210716573, 2021

(CC BY 4.0) | ISSN 2525-3409 | DOI: http://dx.doi.org/10.33448/rsd-v10i7.16573

Tabela 5. Proposta de protocolo de solicitação de exames de imagens odontológicas em casos de Prótese.

\begin{tabular}{|c|c|c|c|c|c|c|c|}
\hline \multicolumn{8}{|c|}{ Casos de prótese } \\
\hline $\begin{array}{c}\text { Hipótese Diagnóstica / } \\
\text { Indicação }\end{array}$ & Idade & $\begin{array}{c}\text { Exame clínico } \\
\text { prévio }\end{array}$ & $\begin{array}{l}\text { Radiografia } \\
\text { periapical }\end{array}$ & $\begin{array}{c}\text { Radiografia } \\
\text { interproximal }\end{array}$ & $\begin{array}{l}\text { Levantamento } \\
\text { periapical }\end{array}$ & $\begin{array}{l}\text { Radiografia } \\
\text { panorâmica }\end{array}$ & Autores \\
\hline \multirow{5}{*}{ Desajustes em próteses fixas } & Grupo 1 & \multirow{4}{*}{ Sim } & \multirow{4}{*}{ Até 2 quadrantes } & \multirow{4}{*}{ Não aplicável } & \multirow{4}{*}{$\begin{array}{l}\text { Acima de } 3 \\
\text { quadrantes }\end{array}$} & \multirow{4}{*}{ Não aplicável } & \multirow{5}{*}{ Liedke, G. S. et al, 2014} \\
\hline & Grupo 2 & & & & & & \\
\hline & Grupo 3 & & & & & & \\
\hline & Grupo 4 & & & & & & \\
\hline & Grupo 5 & \multicolumn{5}{|c|}{ Não aplicável } & \\
\hline \multirow{5}{*}{ Restaurações subgengivais } & Grupo 1 & \multirow{4}{*}{ Sim } & \multirow{4}{*}{ Até 2 quadrantes } & \multirow{4}{*}{ Não aplicável } & \multirow{4}{*}{$\begin{array}{l}\text { Acima de } 3 \\
\text { quadrantes }\end{array}$} & \multirow{4}{*}{ Não aplicável } & \multirow{5}{*}{$\begin{array}{l}\text { Sariyilmaz, E. et al, 2016; } \\
\text { Tsiklakis, K. et al, } 2019\end{array}$} \\
\hline & Grupo 2 & & & & & & \\
\hline & Grupo 3 & & & & & & \\
\hline & Grupo 4 & & & & & & \\
\hline & Grupo 5 & \multicolumn{5}{|c|}{ Não aplicável } & \\
\hline \multirow{5}{*}{$\begin{array}{l}\text { Desadaptações na interface } \\
\text { implante/componente } \\
\text { protético/integridade peri- } \\
\text { implantar }\end{array}$} & Grupo 1 & \multirow{2}{*}{\multicolumn{5}{|c|}{ Não aplicável }} & \multirow{5}{*}{$\begin{array}{l}\text { Liedke, G. S. et al, 2014; } \\
\text { Correia-Sousa, J. et al, } \\
\text { 2015; Sarivilmaz, E. et al, } \\
\text { 2016; Castro, M. F. O. M. } \\
\text { et al, 2017 }\end{array}$} \\
\hline & Grupo 2 & & & & & & \\
\hline & Grupo 3 & \multirow{3}{*}{ Sim } & \multirow{3}{*}{ Até 2 quadrantes } & \multirow{3}{*}{ Não aplicável } & \multirow{3}{*}{$\begin{array}{l}\text { Acima de } 3 \\
\text { quadrantes }\end{array}$} & \multirow{3}{*}{ Não aplicável } & \\
\hline & Grupo 4 & & & & & & \\
\hline & Grupo 5 & & & & & & \\
\hline
\end{tabular}

Fonte: Autores. 


\section{Conclusão}

1. É necessária a correta observação da história pregressa e a execução do exame clínico detalhado para definir se os exames complementares de imagem são necessários e, nesse caso, qual o exame mais indicado, considerando critérios de odontologia baseada em evidências e consenso científico.

2. Critérios de situação clínica e faixa etária são itens importantes para seleção de exames por imagem em odontologia.

3. A proposta de protocolo de solicitação de exames de imagem em odontologia do presente trabalho auxiliará na confecção de diretrizes de utilização para reforçar a necessidade de justificativa para todos os exames de radiografia odontológica realizados.

4. A variabilidade de solicitações de radiografias, particularmente por ser considerado um exame complementar ao diagnóstico inicial, requer dos cirurgiões-dentistas uma avaliação criteriosa para a sua indicação, correlacionando cada tipo de exame a situação clínica de seus pacientes, evitando exposições e gastos desnecessárias.

\section{Referências}

Agência Nacional de Saúde Suplementar. (2018). Projeto Sorrir, Protocolos Módulo: Radiologia. http://ans.gov.br/images/ stories/gestao_em_saude/projeto_sorrir/projeto-sorrir-protocolo-radiologia.pdf

Agência Nacional de Vigilância Sanitária. (2019). Resolução - RDC $N^{O}$ 330, de 20 de dezembro de 2019. Brasil, Ministério da Saúde. https://www.in.gov.br/web/dou/-/resolucao-rdc-n-330-de-20-de-dezembro-de-2019-235414748?inheritRedirect=true

Alves, W. A., Camelo, C. A. C., Guaré, R. O., Costa, C. H. M., \& Almeida, M. S. C. (2014). Proteção radiológica: conhecimento e métodos dos cirurgiõesdentistas. Arquivos em Odontologia, 52(3), 130-135.

American Dental Association. (2012). Dental Radiographic Examinations: Recommendations for patient selection and limiting radiation exposure. U.S. Department of Health and Human Services, National Institutes of Health. https://www.fda.gov/media/84818/download

Beneyto, Y. M., Baños, M. A., Lajarín, L. P., \& Rushton, V. E. (2007). Clinical justification of dental radiology in adult patients: A review of the literature. Medicina Oral, Patologia Oral, Cirurgia Bucal, 12, 244-251.

Caballero, A. D., Arenas, Y. H., \& Acosta, S. M. (2017). Concordancia inter-examinador en la evaluación de hallazgos periodontales mediante radiografías panorâmicas. Revista Odontológica Mexicana, 21(2), 98-102.

Candeiro, G. T. M., Bringel, A. S. F., \& Vale, I. S. (2009). Radiologia digital: revisão de literatura. Revista Odontológica de Araçatuba, 30(2), 38-44.

Capote, T. S. O., Gonçalves, M. A., Gonçalves, A., \& Gonçalves, M. (2005). Panoramic Radiography — Diagnosis of Relevant Structures That Might Compromise Oral and General Health of the Patient. In Emerging Trends in Oral Health Sciences and Dentistry (pp 733-762). Mandeep Singh Virdi.

Castro, M. F. O. M., Coutinho, M. H., Darós, P., Carneiro, V. C., \& de-Azevedo-Vaz, S. L. (2017). Métodos de exames por imagem utilizados no diagnóstico de desadaptação entre implante e componente protético: uma revisão de literatura. Revista Brasileira de Odontologia, 74(2), 162-166.

Cerri1, A., Guarim, J. A., \& Genovese, W. J. (2015). Planejamento e diagnóstico em Odontologia com os princípios bioéticos. Revista da Associação Paulista de Cirurgiões Dentistas, 69(3), 217-225.

Correia-Sousa, J., Madureira, A. R., Carvalho, M. F., Teles, A. M., \& Pina-Vaz, I. (2015). Apical periodontitis and related risk factors: Cross-sectional study. Revista Portuguesa de Estomatologia, Medicina Dentária e Cirurgia Maxilofacial, 56(4), 226-232.

Diangelis, A. J., Andreasen, J. O., Ebeleseder, K. A., Kenny, D. J., Trope, M., Sigurdsson, A., Andersson, L., Bourguignon, C., Flores, M. T., Hicks, M. L., Lenzi, A. R., Malmgren, B., Moule, A. J., Pohl, Y., \& Tsukibosh, M. (2012). International Association of Dental Traumatology guidelines for the management of traumatic dental injuries: 1. Fractures and luxations of permanent teeth. Dental Traumatology, 28(1), 2-12.

Farman, A. G. (2007). Panoramic Radiology: Role in ADA/FDA Use Guidelines. In Panoramic Radiology (pp.33-40). Springer.

Gil, C., Varoli, F. P., Buscatti, M. Y., Costa, C., \& Oliveira, J. X. (2005). Avaliação do perfil dos pacientes encaminhados para a realização de exames radiográficos odontológicos,considerando gênero e faixa etária no que se refere aos meios de proteção radiológica. Revista do Instituto de Ciências da Saúde, 23(4), 269-274

Hwang, S. Y., Choi, E. S., Kim, Y. S., Gim, B. E., Ha, M., \& Kim, H. Y. (2018). Health effects from exposure to dental diagnostic X-ray. Environmental Health and Toxicology, 33(4).

Kühnisch, J., Anttonen, V., Duggal, M. S., Loizides, M. S., Rajasekharan, S., Sobczak, M., Stratigaki, E., A. J. W. G. V., Aps, J. K. M., Horner, K., \& Tsiklakis, K. (2019). Best clinical practice guidance for prescribing dental radiographs in children and adolescents: an EAPD policy document. European Archives of Paediatric Dentistry, 21(4), 375-386.

Liedke, G. S., Spin-Neto, R., Da Silveira H. E. D., \& Wenzel A. (2014). Radiographic diagnosis of dental restoration misfit: a systematic review. Journal of Oral Rehabilitation, 41(12), 957-967. 
Marsi, G, Mengue, A. C., Bertini, F., Cabral, L. A. G., \& Almeida, J. D. (2009). Avaliação da importância do exame clínico para os alunos do curso de graduação da Faculdade de Odontologia de São José dos Campos - UNESP. Revista da ABENO, 9(1), 5-10.

Mendes, F. M.; Pontes, L. R. A., Gimenez, T., Lara, J. S., Camargo, L. B., Michel-Crosato, E., Panutti, C. M., Raggio, D. P., Braga, M. M., \& Novaes, T. F. (2016). Impacto $f$ the radiographic examination on diagnonis and treatment decision of caries lesions in primary teeth - the Caries Detection in Children (CARDEC-01) trial: study protocol for a randomized controlled trial. Trials. 9, 17-69.

Ministério da Saúde. (2008) Manual de especialidades em saúde bucal. Secretaria de Atenção à Saúde. Departamento de Atenção Básica. http://bvsms.saude.gov.br/bvs/publicacoes/manual_especialidades_saude_bucal.pdf

Morais, E. F., Lira, J. A. S., Macedo, R. A. P., Santos, K. S., Elias, C. T. V., \& Morais, M. L. S. A. (2014). Oral manifestations resulting from chemotherapy in children with acute lymphoblastic leucemia. Brazilian Journal of Otorhinolorayngology, 80(1), 78-85.

Moura, L. B., Blasco, M. A. P., \& Damian, M. F. (2014). Exames radiográficos solicitados no atendimento inicial de pacientes em uma Faculdade de Odontologia brasileira. Revista de Odontologia da UNESP, 43(4), 252-257.

Oliveira, M. V., Silva, M. B. F., Junqueira, J. L. C., \& Oliveira, L. B. (2012). Evaluation of the level of knowledge of dental surgeons regarding radiographic techniques, radioprotection measures, and biosafety in Montes Claros, MG Brazil. Arquivos em Odontologia, 48(2), 82-88.

Pontes, L. R. A. (2020). Impacto do exame radiográfico no diagnóstico e decisão de tratamento de lesões de cárie em entes decíduos - CARies DEtection in Children (CARDEC-01) trial. [Doctoral dissertation, São Paulo University]. https://doi.org/10.11606/T.23.2020.tde-26022021-073525

Rushton, V. E., Horner, K., \& Worthington, H. V. B. D. J. (2001). Screening panoramic radiology of adults in general dental practice: radiological findings by V. E. Rushton, K. Horner and H. V. British Dental Journal, 190, 495-501.

Santos, K. C. P., Oliveira, A. S., Hesse, D., Buscatti, M. Y., \& Oliveira, J. X. (2007). Avaliação de radiografias panorâmicas objetivando o cotejamento entre os motivos da solicitação e eventuais achados radiográficos. Revista do Instituto de Ciências da Saúde, 25(4), 419-422.

Sariyilmaz, E., Keskin, C., \& Ozcan, O. (2016). Retrospective analysis of post-treatment apical periodontitis and quality of endodontic treatment and coronal restorations in an elderly Turkish population. Journal of Clinical Gerontology \& Geriatrics, 7, 17-20.

Silva, A. V. C., Pereira, J. R. D., Beatrice, L. C. S., \& Silva, C. H. V. (2016). Diagnóstico de lesão de cárie proximal por imagem - Revisão Sistemática. Odontologia Clínico-Científica, 11(1).

Soares, M. Q. S., Castro Jr, R. C., Santos, P. S. S., Capelozza, A. L. A., \& Fischer-Bullen, I. R. R. (2015). Contribuicão da radiografia panorâmica no diagnóstico de calcificação de ateroma de carótida: relato de caso e revisão da literatura. Revista Portuguesa de Estomatologia, Medicina Dentária e Cirurgia Maxilofacial, 56(2), 127-131.

Sousa, A. A., Cheib, P. L., Andrade Júnior, I., Oliveira, D. D., Souki, B. Q., \& Cevidanes, L. H. S. (2016). Comparação de 2 disjuntores na expansão maxilar em pacientes com fenda labiopalatina: relato de 2 casos. Revista Portuguesa de Estomatologia, Medicina Dentária e Cirurgia Maxilofacial, 57(2), 116-124.

Sulimana, B., \& Abdelgadir, A. H. (2018). Patient radiation doses in intraoral and panoramic X-ray examinations in Sudan. Physica Medica, 46, 148-152.

Tsiklakis, K., Mitsea, A., Tsichlaki, A., \& Pandis, N. (2019). A systematic review of relative indications and contraindications for prescribing panoramic radiographs in dental paediatric patients. European Archives of Paediatric Dentistry, 21(4), 387-406.

Van, A. J. W. G., Pauwels, N. S., Cauwels, R. G. E. C., \& Rajasekharan, S. (2020). Outcomes of diferent radioprotective precautions in children undergoing dental radiography: a systematic review. European Archives of Paediatric Dentistry, 21(4), 463-508.

Villoria, E. M., Francio, L. A., Cunha, C. H. R., \& Manzi, F. R (2016). Identification of simulated periapical diseases using five different diagnostic imaging methods. Revista Portuguesa de Estomatologia, Medicina Dentária e Cirurgia Maxilofacial, 57(3), 138-145.

Watanabe, P. C. A., Faria, V., \& Camargo, A. J. (2017). Multiple Radiographic Analysis (Systemic Disease): Dental Panoramic Radiography. Journal of Oral Health and Dental Care, 1(007).

White, S. C., \& Pharoah, M. J (2020). Radiologia Oral - Princípios e Interpretação (8a ed). GEN Guanabara Koogan.

Zavala, C. E. P. S. M., Mendiburu, J. C., \& Lugo-Ancona, P. (2017). Relación entre la oclusión traumática y abfracciones; su rol en las afecciones pulpares. Revista Odontológica Mexicana, 21(2), 81-86. 